\title{
Trastornos musculoesqueléticos en recicladores que laboran en Lima Metropolitana
}

Musculoskeletal disorders among recyclers working in Metropolitan Lima

\author{
Juan Morales-Quispe ${ }^{1,2, a}$, César Abraham Suárez Oré ${ }^{1, b}$, Claudia Paredes Tafur ${ }^{1, c}$, \\ Vilma Mendoza Fasabi ${ }^{1, c}$, Lucero Meza Aguilar ${ }^{1, c}$, Lumy Colquehuanca Huamani, \\ ${ }^{1}$ Facultad de Ciencias de la Salud de la Universidad de Ciencias y Humanidades. \\ ${ }^{2}$ Dirección Regional de Salud del Callao. \\ ${ }^{a}$ Médico cirujano; ${ }^{b}$ Licenciado en enfermería; ${ }^{c}$ Estudiante de enfermería.
}

\begin{abstract}
Resumen
Introducción. Los recicladores en su mayoria desarrollan sus actividades en precarias condiciones sanitarias y de seguridad ocupacional. Los sintomas de los trastornos musculoesqueléticos (TME) es un problema que afecta a todos los sectores y ocupaciones. Objetivos. Conocer la percepción de síntomas de los TME. Diseño. Estudio descriptivo transversal. Lugar. Lima Metropolitana. Participantes. Recicladores de cualquier edad y género. Intervenciones. Aplicación del Cuestionario Nórdico. Principales medidas de resultados. Presencia de sintomas de TME por regiones anatómicas. Resultados. De los 131 participantes, 114 cumplieron con los criterios de inclusión, 58,8\% ( $n=67$ ) fueron varones y 41,2\% ( $n=47)$ mujeres, con una media de 41,2 años ( $D E$ : 14,61; min: 18, máx: 77). La región anatómica con más sintomas fue la región lumbar, y fue la dolencia que más interferencia tuvo con las tareas de la casa 0 el trabajo. En el grupo etario de 20 a 39 años, los síntomas musculoesqueléticos más frecuentes se presentaron en la región cervical $(p=0,012)$, mientras que en los mayores de 50 años se presentaron en las rodillas $(p=0,040)$. Conclusiones. La percepción de sintomas de los TME en los recicladores se presentó predominantemente en la región lumbar y fue la dolencia que más interferencia tuvo con las tareas de casa o el trabajo.
\end{abstract}

Palabras clave. Músculo Esquelético; Dolor Muscular; Ergonomía; Salud Laboral.

\section{Abstract}

Introduction: Recyclers mostly operate in poor sanitary conditions and low occupational safety. The symptoms of musculoskeletal disorders (MSD) represent a problem affecting all the sectors and occupations. Objective. To determine the perception of MSD symptoms. Design. Cross-sectional study. Setting. Metropolitan Lima. Participants. Recyclers of any age and gender. Interventions. Implementation of Nordic Questionnaire. Main outcome measures. Presence of MSD symptoms according to anatomical regions. Results. Out of 131 selected participants, 114 met the inclusion criteria of which $58.8 \%(n=67)$ were male and $41.2 \%(n=47)$ female with an average age of 41.2 years (SD: 14.61; min: 18, max: 77). The anatomical region where most of the symptoms occurred was the lumbar region where the disease interfered with most of the household chores or work. In the age group 20-39 years, the most frequent musculoskeletal symptoms occurred in the cervical region $(p=0.012)$, whereas among people older than 50 years they occurred in the knees $(p=0.040)$. Conclusions. The perception of the symptoms of musculoskeletal disorders among recyclers was predominantly present in the lumbar region and it was the disease that had the most interference with household chores or work.

Keywords. Skeletal Muscle; Muscle Pain; Ergonomics; Occupational Health.

An Fac med. 2016;77(4):357-63 / http://dx.doi.org/10.15381/anales.v77i4.12655

\section{INTRODUCCIÓN}

El Banco Mundial hace referencia que a nivel mundial unas 15 millones de personas se ganan la vida recuperando material reciclable en la basura. De ellas, cuatro millones lo hacen en América Latina, al menos un $75 \%$ de forma insalubre ${ }^{(1)}$. La Evaluación Regional del Manejo de Residuos Sólidos Urbanos en América Latina y el Caribe 2010 (EVAL 2010) estimó que existen un total de 8,57 segregadores por cada 10000 habitantes en América Latina y el Caribe, lo que se traduce en poco más de 400000 personas ${ }^{(2)}$. En el Perú hay más de 100000 recicladores que trabajan en las ciudades de todo el país, 20000 en Lima, y en el Callao se estima que hay 1500 recicladores ${ }^{(3,4)}$. $\mathrm{La}$ Organización Internacional del Trabajo (OIT) señala que el empleo informal está relacionado a malas condiciones laborales, desprotección, inestabilidad y falta de derechos ${ }^{(5)}$. La informalidad y las condiciones precarias de trabajo en que los segregadores generalmente desarrollan sus tareas generan diversos problemas de tipo social, ambiental y de gestión propiamente dichos ${ }^{(2)}$.
En América Latina y el Caribe existen recicladores organizados y no organizados dependiendo si pertenecen o no a una organización, cooperativa, gremio o asociación; la pertenencia les permite tener mayor posibilidad de adicionar valor a los materiales. Los recicladores en su mayoría desarrollan su actividad en precarias condiciones sanitarias, sin ningún tipo de seguridad ocupacional ni vinculación con el sistema de protección social ${ }^{(6)}$. En el Perú, según el Instituto Nacional de Estadística e Informática (INEI), el 40\% de hogares peruanos tiene ingresos laborales 
provenientes íntegramente del empleo en el sector informal ${ }^{(7)}$ y una de las actividades donde tanto hombres y mujeres encuentran una oportunidad de generar ingresos es el reciclaje y en muchos casos involucran familias enteras quienes realizan jornadas largas que superan los estándares laborales y en condiciones no saludables ${ }^{(4)}$.

De acuerdo al Instituto Nacional para la Seguridad y Salud Ocupacional (NIOSH) de los Estados Unidos, un trastorno musculoesquelético relacionado con el trabajo es una lesión de los músculos, tendones, ligamentos, nervios, articulaciones, cartílagos, huesos o vasos sanguíneos de los brazos, las piernas, la cabeza, el cuello o la espalda que se produce o se agrava por tareas laborales, como levantar, empujar o jalar objetos. Los síntomas pueden incluir dolor, rigidez, hinchazón, adormecimiento y cosquilleo ${ }^{(8)}$.

La I Encuesta Centroamericana de Condiciones de Trabajo y Salud aplicada a los trabajadores insertas en la economía formal e informal muestra que las regiones cervical y dorsal presentaron las prevalencias de dolor musculoesquelético (DME) más altas, por encima del 30\% en todos los casos; la prevalencia en las mujeres fue superior a la de los hombres ${ }^{(9)}$. El dolor musculoesquelético es un problema que afecta de forma creciente y universal a todos los sectores y ocupaciones, y que puede desencadenarse por una compleja red de factores de riesgo. Las personas con dolor intenso reducen su rendimiento laboral seis veces más que las personas que no padecen dolor o lo padecen leve o moderada. Además, el dolor intenso se sitúa como el principal motivo de absentismo laboral ${ }^{(10)}$. En el Perú, según el Instituto Nacional de Rehabilitación, en el Primer trimestre de 2014, de todas las deficiencias atendidas en consulta externa, el 25,8\% fue de origen musculoesquelético ${ }^{(11)}$, mientras que para el Ministerio de Trabajo y Promoción del Empleo en el 2014, 29,98\% de la notificación de enfermedades ocupacionales fue de origen musculoesquelética ${ }^{(12)}$.
Del total de la población peruana, Lima y Callao concentran la mayor proporción, donde $60 \%$ de la población ocupada se encuentra en el sector infor$\mathrm{mal}{ }^{(13)}$; son las regiones que producen el mayor volumen de residuos sólidos y es una fuente de empleo para muchos que no tienen acceso al empleo formal. Dado que es una población importante cuya labor contribuye al cuidado de la ciudad y el medio ambiente, haciendo uso de elementos básicos como herramientas de trabajo, se exponen a un conjunto de riesgos, entre ellos los de origen ergonómico. Por tanto, el objetivo del presente estudio fue conocer la percepción de síntomas de los trastornos musculoesqueléticos según las regiones anatómicas y si estos síntomas interfieren con las tareas del hogar y el trabajo de los recicladores que laboran en los distritos de Lima Metropolitana.

\section{MÉTODOS}

En el presente estudio descriptivo transversal participaron recicladores que laboraban en la vía pública de la jurisdicción de los distritos de Lima Metropolitana: Cercado de Lima, Santa Anita, San Juan de Lurigancho, Independencia, San Martín de Porres, Los Olivos, Comas y Carabayllo. La mayor parte de los participantes en el estudio fueron los recicladores que trabajaban en la jurisdicción de los distritos de Lima Norte. La recolección de datos se llevó a cabo entre noviembre y diciembre de 2015.

Los criterios de inclusión fueron: toda persona de cualquier edad y género dedicada al reciclaje de cualquier residuo. Los participantes fueron sujetos con integridad en el aparato locomotor, con suficiente capacidad para establecer comunicación por medio verbal o escrito y dispuesto a participar en el estudio de forma voluntaria y previo registro de la firma en el formato del consentimiento informado. Se excluyó del estudio a los sujetos que no lograron completar la información solicitada y a los que no firmaron el consentimiento informado.
La selección de la muestra se realizó por muestreo no probabilístico de tipo muestreo por conveniencia, considerándose a los recicladores fácilmente accesibles; y el tamaño de la muestra estuvo constituido por la cantidad obtenida dentro del periodo planificado para la recolección de datos, entre noviembre y diciembre de 2015.

La variable de estudio fue la percepción de los síntomas de los trastornos musculoesqueléticos en los recicladores. Para su evaluación se empleó el Cuestionario Nórdico de Kuorinka (NMQ). El cuestionario no pretende dar un diagnóstico clínico, sino como un tamizaje de los desórdenes musculoesqueléticos en el contexto ergonómico, los cuales pueden servir como una herramienta de diagnóstico del entorno laboral ${ }^{(14)}$. El cuestionario proporciona información sobre los síntomas musculoesqueléticos en diversas áreas del cuerpo ocurridos en el pasado, los últimos 12 meses hasta los 07 días previos $^{(14)}$. La versión española posee los coeficientes de consistencia y fiabilidad entre 0,727 y $0,816^{(15)}$. El NMQ se ha aplicado a una amplia gama de grupos ocupacionales para evaluar los problemas músculoesqueléticos ${ }^{(16)}$. Antes del empleo del instrumento, los investigadores han evaluado la comprensión de los términos usados en el cuestionario y se adjuntó una representación gráfica de los segmentos corporales. La versión final del instrumento fue revisado por un médico ocupacional sin vínculo al equipo de investigación y fue calificado como instrumento adecuado.

La técnica empleada para la recolección de información fue la encuesta, utilizando un cuestionario como instrumento. La aplicación del instrumento estuvo a cargo de estudiantes de enfermería previamente capacitados en el manejo del instrumento. El periodo de aplicación fue entre noviembre y diciembre de 2015, a todos los sujetos que cumplieron con los criterios de inclusión y que al momento de la aplicación del instrumento se encontraban realizando labores de reciclaje en el domicilio, local de acopio o vía pública en 
la jurisdicción de cualquier distrito de Lima Metropolitana y con fácil acceso a los encuestadores.

El análisis estadístico se realizó empleando la versión 20 del IBM SPSS. Se determinó las frecuencias absolutas y relativas de las características sociodemográficas, características laborales, los factores y eventos relacionados con el trabajo, la edad media y la desviación estándar, los valores mínimos y máximos tanto para la edad como para el número de hijos. Se clasificó los síntomas durante los últimos 12 meses, durante los últimos 07 días y según la interferencia con las tareas de casa o el trabajo. Se realizó el análisis bivariado entre las variables de interés; para establecer la asociación se empleó la prueba no paramétrica chi cuadrado $\left(\mathrm{X}^{2}\right)$ y su correspondiente p-valor. Se concluyó con la elaboración de tablas y gráficos.

La participación de los sujetos fue voluntaria y, antes de la aplicación del instrumento, los participantes firmaron el formato de consentimiento informado. El proyecto fue aprobado por la dirección de investigación de la Universidad de Ciencias y Humanidades.

\section{RESULTADOS}

De un total de 131 participantes seleccionados, 17 individuos fueron excluidos, nueve por no contar con la firma del consentimiento informado, tres por no haber completado el llenado de la encuesta y cinco rechazaron participar en el estudio. La muestra quedó constituida por 114 individuos, de los cuales $58,8 \%(\mathrm{n}=67)$ eran varones y $41,2 \%(n=47)$ mujeres. Según el grupo de edad, $2,6 \%(n=3)$ fue menor de 20 años, $48,2 \%(\mathrm{n}=55)$ tenía entre $20 \mathrm{y}$ 39 años, $19,3 \%(n=22)$ entre 40 y 49 años y 29,8\% (n=34) 50 a más años de edad. La edad media fue 41,22 años (DE: 14,61, Min: 18, Max: 77). El estado civil predominante fue soltero(a) con 33,3\% ( $\mathrm{n}=38)$, el grado de instrucción predominante fue el nivel secundaria con $53,5 \%(n=61) ; 66,7 \%$ $(\mathrm{n}=76)$ manifestó tener hijos (pro-
Tabla 1. Características sociodemográficas de los recicladores.

\begin{tabular}{|c|c|c|}
\hline Características sociodemográficas & $\mathrm{n}$ & Porcentaje \\
\hline Total & 114 & 100 \\
\hline \multicolumn{3}{|l|}{ Sexo } \\
\hline Masculino & 67 & 58,8 \\
\hline Femenino & 47 & 41,2 \\
\hline \multicolumn{3}{|l|}{ Edad (años) } \\
\hline$<20$ & 3 & 2,6 \\
\hline $20-39$ & 55 & 48,2 \\
\hline $40-49$ & 22 & 19,3 \\
\hline $50 a+$ & 34 & 29,8 \\
\hline Media & 41,22 (DE:14,61) & $\operatorname{Min}=18, \operatorname{Max}=77$ \\
\hline \multicolumn{3}{|l|}{ Estado civil } \\
\hline Soltero(a) & 38 & 33,3 \\
\hline Conviviente & 36 & 31,6 \\
\hline Casado(a) & 27 & 23,7 \\
\hline Divorciado(a) / separado(a) & 11 & 9,6 \\
\hline Viudo(a) & 2 & 1,8 \\
\hline \multicolumn{3}{|l|}{ Grado de instrucción } \\
\hline Primaria & 31 & 27,2 \\
\hline Secundaria & 61 & 53,5 \\
\hline Superior incompleto & 9 & 7,9 \\
\hline Superior completo & 13 & 11,4 \\
\hline \multicolumn{3}{|l|}{ Tenencia de hijo } \\
\hline Sí & 76 & 66,7 \\
\hline No & 38 & 33,3 \\
\hline Promedio de hijos & 2 & $\operatorname{Min}=1, \operatorname{Max}=8$ \\
\hline \multicolumn{3}{|l|}{ Región de nacimiento } \\
\hline Lima & 57 & 50 \\
\hline Callao & 5 & 4,4 \\
\hline Costa (excepto Lima y Callao) & 13 & 11,4 \\
\hline Sierra & 27 & 23,7 \\
\hline Selva & 12 & 10,5 \\
\hline
\end{tabular}

medio $=2, \min =1, \max =8)$. Según la región de nacimiento, 54,4\% $(n=62)$ fueron de Lima y Callao y el resto de otras ciudades de las tres regiones del país, la mayoría de la región sierra. Tabla 1.

En relación al tiempo de servicio como reciclador, $28,9 \%(n=33)$ tenía entre 3 a 5 años, $13,2 \%(n=15)$ llevaba más de 10 años dedicándose a la actividad, mientras que $16,7 \%(n=19)$ tenía menos de un año. El 38,6\% ( $\mathrm{n}=44)$ mencionó tener otro empleo paralelo.
La mayoría dedicaba 4 a 6 días de la semana $(38,6 \%)$, trabajaba entre 6 y 8 horas diarias $(36,0 \%)$ y predominantemente en los turnos mañana y tarde $(63,2 \%)$. El 86\% $(n=86)$ refirió agrado por el trabajo que realizaba, mientras que la percepción de agrado a nivel de la familia fue de $61,4 \%(n=70)$. Tabla 2.

Entre los factores individuales de los recicladores, en relación al dominio de los miembros superiores, el $67,5 \%$ $(n=77)$ era diestro, 42,1\% $\quad(n=48)$ 
Tabla 2. Características sociodemográficas de los recicladores.

\begin{tabular}{|c|c|c|}
\hline Características laborales & $\mathrm{n}$ & Porcentaje \\
\hline \multicolumn{3}{|l|}{ Tiempo de servicio (años) } \\
\hline$<1$ & 19 & 16,7 \\
\hline $1-2$ & 24 & 21,1 \\
\hline $3-5$ & 33 & 28,9 \\
\hline $5-10$ & 23 & 20,2 \\
\hline$>10$ & 15 & 13,2 \\
\hline \multicolumn{3}{|c|}{ Tenencia de otro empleo paralelo } \\
\hline Sí & 44 & 38,6 \\
\hline No & 70 & 61,4 \\
\hline \multicolumn{3}{|l|}{ Días de trabajo por semana } \\
\hline 1-3 días/sem & 30 & 26,3 \\
\hline 4-6 días/sem & 44 & 38,6 \\
\hline Todos los días & 40 & 35,1 \\
\hline \multicolumn{3}{|l|}{ Horas de trabajo al día } \\
\hline 1-5 horas/día & 36 & 31,6 \\
\hline 6-8 horas/día & 41 & 36,0 \\
\hline 9-12 horas/día & 27 & 23,7 \\
\hline 13-15 horas/día & 9 & 7,9 \\
\hline$>15$ horas/día & 1 & 0,9 \\
\hline \multicolumn{3}{|l|}{ Turnos de trabajo } \\
\hline Mañana & 25 & 21,9 \\
\hline Mañana-tarde & 72 & 63,2 \\
\hline Mañana-tarde-noche & 17 & 14,9 \\
\hline \multicolumn{3}{|c|}{ Agrado personal por el trabajo } \\
\hline Sí & 86 & 75,4 \\
\hline No & 28 & 24,6 \\
\hline \multicolumn{3}{|l|}{ Agrado familiar por el trabajo } \\
\hline Sí & 70 & 61,4 \\
\hline No & 44 & 38,6 \\
\hline
\end{tabular}

empleaba elementos de protección personal durante el trabajo, siendo los guantes referidos en la mayoría de los casos, seguido por los elementos de protección buco nasal. El 26,3\% $(n=30)$ recibió atención médica en los últimos 12 meses; 22,8\% $(n=26)$ refirió haber tenido algún tipo de accidente durante el trabajo en los últimos 12 meses, siendo las caídas y contusiones las más frecuentes, seguido por las heridas cortantes producidas al manipular los objetos que reciclaban. Tabla 3 .

Respecto a la presencia de síntomas musculoesqueléticos, el dolor, molestias afectado. La región dorsal, con 43,9\% $(n=50)$, en $26,3 \%(n=30)$ interfirió con sus labores y en $28,1 \% \quad(n=32)$ tuvo síntomas durante los últimos siete días. Tabla 4. Según el género, tanto en los varones como en las mujeres, la región anatómica que más síntomas presentaron fue la región lumbar, mientras que el codo fue la región anatómica con menos síntomas. Figura 1.

La presencia de dolor, molestias o incomodidad a nivel de la región cervical durante los últimos 12 meses, estuvo presente en mayor proporción en el grupo de edad de 20 a 39 años $(\mathrm{X} 2=10,927 ; \mathrm{p}=0,012)$, mientras que dichos síntomas a nivel de las rodillas estuvo presente en mayor proporción en los trabajadores de 50 a más años de edad $(X 2=8,295 ; \mathrm{p}=0,040)$. También hubo diferencias estadísticamente significativas entre la presencia de síntomas a nivel de la región lumbar durante los últimos 12 meses y el dominio diestro de los miembros superiores $(\mathrm{X} 2=6,325 ; \mathrm{p}=0,042)$, y en los recicladores que laboraban en los turnos mañana y tarde $(\mathrm{X} 2=6,975 ; \mathrm{p}=0,031)$, mientras que los síntomas a nivel de los tobillos se presentaron en mayor proporción en trabajadores con grado de instrucción primaria $(\mathrm{X} 2=8,260$; $\mathrm{p}=0,041)$. No se encontró diferencias significativas entre el género, tiempo de servicio, días de trabajo por semana ni con las horas de trabajo al día. Tabla 5.

\section{DISCUSIÓN}

En el estudio se halló personas dedicadas al reciclaje desde adolescentes hasta mayores de 50 años; buena parte eran migrantes y la mayoría procedía de la región sierra, con nivel de instrucción entre primaria y secundaria. El crecimiento de la ciudad de Lima responde a la migración interna que han constituido los nuevos distritos que cuentan con múltiples asentamientos humanos, con familias en condiciones precarias y con limitaciones para acceder a empleos con remuneraciones suficientes; así, optan por la actividad económica informal y dentro de ella el reciclaje. 


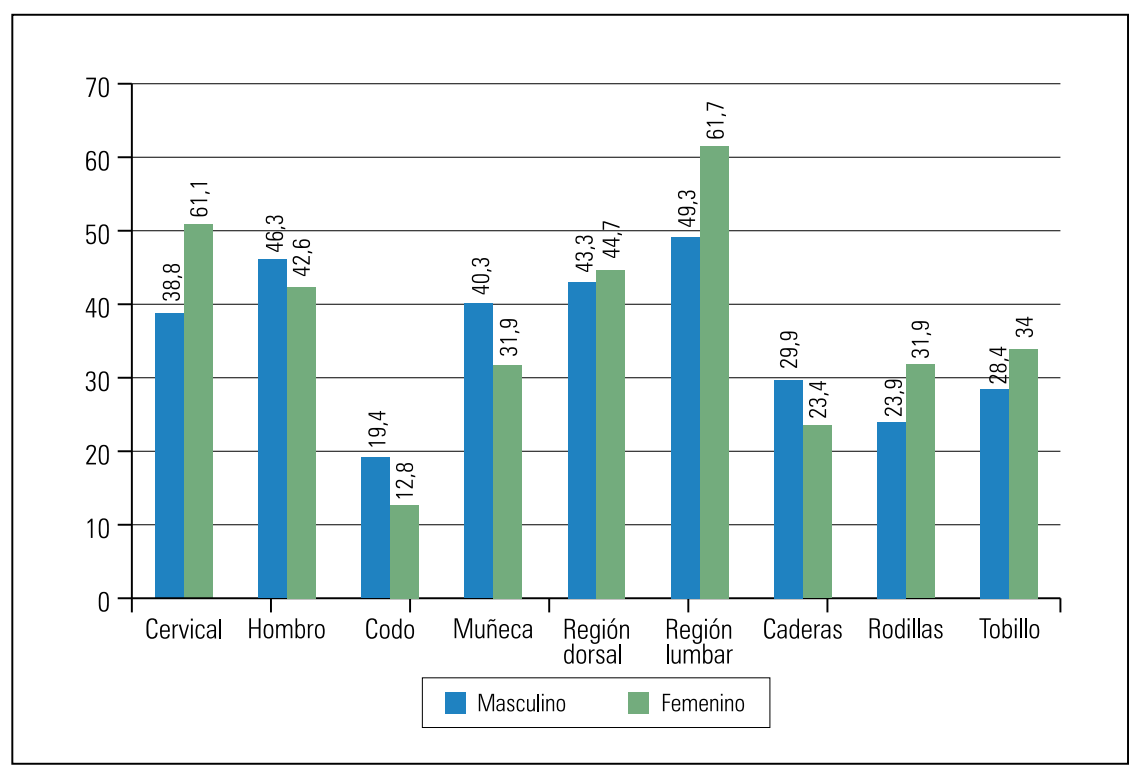

Figura 1. Percepción de síntomas musculoesqueléticos sedún la región anatómica y género de los recicladores.

Tal como ocurre en otros países, el movimiento de pequeñas a grandes ciudades se da en individuos de menor edad, menor nivel educativo y que consiguen menores remuneraciones ${ }^{(17)}$. Si bien en nuestro estudio los recicladores en su

mayoría declaró tener el nivel de educación secundaria, seguido por los que poseían educación primaria, fue en menor proporción a lo hallado en México, donde 52\% afirmó tener solo educación primaria y $23 \%$ educación secundaria,

Tabla 3. Factores y eventos relacionados con el trabajo.

\begin{tabular}{lcc} 
Factores y eventos relacionado con el trabajo & $n$ & Porcentaje \\
Dominio de los miembros superiores & 77 & 67,5 \\
Diestro & 10 & 8,8 \\
Zurdo & 27 & 23,7 \\
Ambidiestro & & \\
Uso de elementos de protección personal & 48 & 42,1 \\
Sí & 66 & 57,9 \\
No & & \\
Atención médica en los últimos 12 meses & 30 & 26,3 \\
Sí & 84 & 73,7 \\
No & & \\
Ocurrencia de accidentes en los últimos 12 meses & 26 & 22,8 \\
Sí & 88 & 77,2 \\
No & & \\
\hline Principales accidentes & 10 & 8,8 \\
Caída y contusiones & 7 & 6,1 \\
Herida cortante & 3 & 2,6 \\
Mordedura canina & 4 & 0,9 \\
Accidente de tránsito & 1 & \\
Mordedura de araña & & \\
\hline
\end{tabular}

aunque se trató de 'pepenadores', que son recolectores de residuos sólidos en los tiradores de basura y, en su mayoría, migrantes de zonas rurales pobres con poca experiencia laboral y con ingresos monetarios precarios ${ }^{(18)}$. En Buenos Aires, Argentina, los recicladores informales (cartoneros) tienen niveles de escolaridad bajos en comparación con la población argentina ${ }^{(19)}$.

Los síntomas musculoesqueléticos estuvieron presentes en todas las regiones anatómicas y en ambos sexos, siendo la región lumbar en mayor proporción, seguido por la región de los hombros, región cervical y dorsal, que estuvieron presentes en más del 50\% de los trabajadores, mientras que los síntomas en el resto de las regiones anatómicas estuvieron presentes en menos del 50\% de los trabajadores. En Londrina, Brasil, la presencia de dolor musculoesquelético fue referido por todos los recicladores participantes; hubo aumento significativo de dolor en los miembros superiores, dolor cervical y en los miembros inferiores después del inicio de la actividad; el dolor lumbar y dorsal no ha mostrado empeoramiento después de la actividad ${ }^{(20)}$.

En el presente trabajo, el análisis bivariado muestra que los síntomas musculoesqueléticos durante los últimos 12 meses a nivel de la región cervical estuvo presente en mayor proporción entre los 20 y 39 años; los síntomas en las rodillas estuvo en mayor proporción en recicladores que tenían entre 50 y más años de edad; los síntomas en la región lumbar se presentaron en mayor proporción en trabajadores diestros y en los que laboraban en los turnos mañana-tarde; mientras que los síntomas a nivel de los tobillos se presentaron en mayor proporción en los trabajadores con grado de instrucción primaria. Los recicladores pasan largas horas caminando y llevando consigo entre los hombros los costales que contienen los materiales obtenidos o en otros casos haciendo uso de vehículos no motorizados, lo que termina generando síntomas. En Londrina, gran parte de los recicladores permanecen de pie y car- 
Tabla 4. Percepción de síntomas musculoesqueléticos según la región anatómica.

\begin{tabular}{|c|c|c|c|c|c|c|}
\hline \multirow[t]{2}{*}{ Región anatómica } & \multicolumn{2}{|c|}{$\begin{array}{c}\text { Dolor, molestias o } \\
\text { incomodidad en los } \\
\text { últimos } 12 \text { meses }\end{array}$} & \multicolumn{2}{|c|}{$\begin{array}{c}\text { Interferencia con } \\
\text { las tareas de casa o } \\
\text { el trabajo debido al } \\
\text { problema }\end{array}$} & \multicolumn{2}{|c|}{$\begin{array}{l}\text { Presencia de problemas } \\
\text { durante los últimos } \\
\text { siete días } \\
\end{array}$} \\
\hline & $\mathrm{n}$ & $\%$ & $\mathrm{n}$ & $\%$ & $n$ & $\%$ \\
\hline Cuello & 50 & 43,9 & 31 & 27,2 & 21 & 18,4 \\
\hline Hombros & 51 & 44,7 & 28 & 24,6 & 21 & 18,4 \\
\hline Derecho & 26 & 22,8 & & & & \\
\hline Izquierdo & 8 & 7,0 & & & & \\
\hline Ambos & 17 & 14,9 & & & & \\
\hline Codos & 19 & 16,7 & 14 & 12,3 & 8 & 7,0 \\
\hline Derecho & 9 & 7,9 & & & & \\
\hline Izquierdo & 4 & 3,5 & & & & \\
\hline Ambos & 6 & 5,3 & & & & \\
\hline Muñecas / manos & 42 & 36,9 & 25 & 21,9 & 15 & 13,2 \\
\hline Derecho & 23 & 20,2 & & & & \\
\hline Izquierdo & 6 & 5,3 & & & & \\
\hline Ambos & 13 & 11,4 & & & & \\
\hline Región dorsal & 50 & 43,9 & 30 & 26,3 & 32 & 28,1 \\
\hline Región lumbar & 62 & 54,4 & 52 & 45,6 & 43 & 37,7 \\
\hline Caderas / nalgas & 31 & 27,2 & 26 & 22,8 & 20 & 17,5 \\
\hline Rodillas & 31 & 27,2 & 22 & 19,3 & 23 & 20,2 \\
\hline Tobillos / pies & 35 & 30,7 & 27 & 23,7 & 23 & 20,2 \\
\hline
\end{tabular}

gan peso la mayor parte de la jornada de trabajo ${ }^{(20)}$.

Toda actividad laboral implica exposición a un conjunto de riesgos y peligros, y considerando que menos de 50\% de los trabajadores refiere hacer uso de algún elemento de protección personal consistente en guantes y mascarilla, termina generando daños a la salud. En nuestra población de estudio, las caídas y contusiones fueron informadas con más frecuencia, seguido por las heridas cortantes ocasionadas al hurgar y manipular los residuos; asimismo, fueron víctimas de mordedura canina y accidente de tránsito mientras realizaban su trabajo. En Argentina, el 46\% de los encuestados informó que se había cortado en una pieza de metal, $58 \%$ con vidrio. Entre los problemas de salud relacionados con el reciclaje informal se describen los accidentes de tráfico, cortes de vidrio y metal, agotamiento, fatiga, quemaduras, dolor y molestias (dolor de cuerpo, dolor de cabeza, dolor de oído y artritis), problemas dermatológicos, respiratorios e infecciones ${ }^{(19)}$.

Los resultados presentados deben interpretarse considerando las siguientes limitaciones: no se incluyó la totalidad de los distritos pertenecientes a Lima Metropolitana. Además, tanto la selección de los distritos como los sujetos participantes se realizó por conveniencia sobre la base de criterios de accesibilidad a los encuestadores. El hecho de ser un diseño transversal, emplear un cuestionario y tomar parte del tiempo dedicado el trabajo de los recicladores, más aún, tratándose de un empleo que genera algún grado de estigma, pudo haber influido en la honestidad de algunas respuestas.

En suma, en los recicladores informales de Lima Metropolitana, la región anatómica que más síntomas presentó en los 12 últimos meses y los últimos siete días fue la región lumbar, y fue la dolencia que más interferencia tuvo con las tareas de casa o el trabajo. Entre los factores asociados a la percepción de síntomas en la región lumbar estu-

Tabla 5. Percepción de sintomas musculoesqueléticos según la región anatómica y grupos de edad de los recicladores.

\begin{tabular}{|c|c|c|c|c|c|c|c|c|c|c|c|}
\hline \multirow{2}{*}{ Región anatómica } & \multicolumn{2}{|c|}{$<20$ años } & \multicolumn{2}{|c|}{ 20-39 años } & \multicolumn{2}{|c|}{ 40-49 años } & \multicolumn{2}{|c|}{$50 a+$} & \multicolumn{2}{|c|}{ Total } & \multirow{2}{*}{ Valor $p$} \\
\hline & $\mathrm{n}$ & $\%$ & $n$ & $\%$ & $\mathrm{n}$ & $\%$ & $\mathrm{n}$ & $\%$ & $\mathrm{n}$ & $\%$ & \\
\hline Cervical & 0 & 0,0 & 30 & 54,5 & 4 & 18,2 & 16 & 47,1 & 50 & 43,9 & 0,012 \\
\hline Hombro & 2 & 66,7 & 29 & 52,7 & 9 & 40,9 & 11 & 32,4 & 51 & 44,7 & 0,236 \\
\hline Codo & 1 & 33,3 & 10 & 18,2 & 2 & 9,1 & 6 & 17,6 & 19 & 16,7 & 0,654 \\
\hline Muñeca & 1 & 33,3 & 23 & 41,8 & 8 & 36,4 & 10 & 29,4 & 42 & 36,8 & 0,703 \\
\hline Región dorsal & 2 & 66,7 & 28 & 50,9 & 9 & 40,9 & 11 & 32,4 & 50 & 43,9 & 0,302 \\
\hline Región lumbar & 1 & 33,3 & 28 & 50,9 & 13 & 59,1 & 20 & 58,8 & 62 & 54,4 & 0,736 \\
\hline Caderas & 1 & 33,3 & 17 & 30,9 & 6 & 27,3 & 7 & 20,6 & 31 & 27,2 & 0,755 \\
\hline Rodillas & 0 & 0,0 & 10 & 18,2 & 6 & 27,3 & 15 & 44,1 & 31 & 27,2 & 0,040 \\
\hline Tobillos / pies & 0 & 0,0 & 19 & 34,5 & 6 & 27,3 & 10 & 29,4 & 35 & 30,7 & 0,602 \\
\hline Total & 3 & 100 & 55 & 100 & 22 & 100 & 34 & 100 & 114 & 100 & \\
\hline
\end{tabular}


vieron el dominio diestro de los miembros superiores y laborar en los turnos mañana y tarde; para la percepción de los síntomas de la región cervical el factor asociado fue el grupo de edad de 20 a 39 años; la percepción de síntomas en las rodillas se asoció al grupo de edad mayor de 50 años; y la percepción de síntomas a nivel de los tobillos se relacionó al grado de instrucción primaria. No se encontró diferencias significativas entre el género, tiempo de servicio, días de trabajo por semana ni con las horas de trabajo al día.

\section{AGRADECIMIENTOS}

Agradecemos al Dr. Ferdinan Carlos Ayma por su colaboración en la revisión del artículo y a la Lic. Keteryna Kryvoviaz por la revisión del resumen en inglés. A los estudiantes del VII ciclo de la escuela profesional de enfermería de la Universidad de Ciencias y Humanidades, quienes participaron en la recolección de información: Bernaola Valdivia Elizabeth, Hurtado Muñico Katerine, Pinillos Vargas Emily, Cayetano Miranda Lizandra, Céspedes Flores Melchora, Vera Caballero Milagros, Murillo Rosillo Verónica, Seclen Terán Jorge.

\section{REFERENCIAS BIBLIOGRÁFICAS}

1. Banco Mundial. Banco Mundial [Internet]. 2014. Disponible en: http://www.bancomundial.org/es/ news/feature/2014/12/23/de-pobres-a-empresarios-de-la-basura

2. Tello P, Martinez E, Daza D, Soulier M, Terraza $\mathrm{H}$. Informe de la Evaluacion Regional del Manejo de Residuos Solidos Urbanos en America Latina y el Caribe 2010 [Internet]. Organizacion Panamericana de la Salud. 2010. Disponible en: http://idbdocs.iadb.org/wsdocs/getdocument. aspx?docnum $=36466973$
3. Ruiz A, Zela C, Pajuelo M, Roldan P, Rodriguez J. Through Garbage Changing Minds and Hearts [Internet]. 2015. 01-168 p. Disponible en: http:// www.informalwastesector.net/view/article/51cbf $1 \mathrm{e} 27896 \mathrm{bb} 431 \mathrm{f6a} 7190 /$ ?topic $=51 \mathrm{cbfc} 95 \mathrm{f} 702 \mathrm{fc}$ 2ba812e4a4

4. Ministerio del Ambiente. Informe Anual de Residuos Sólidos Municipales y No Municipales en el Perú, Gestión 2009 [Internet]. 2010. Disponible en: http://www.redrrss.pe/material/20110601095917. pdf

5. Organización Internacional del Trabajo. Panorama Laboral 2014 América Latina y el Caribe [Internet]. 2014. Disponible en: http://www.ilo.org/wcmsp5/ groups/public/---americas/---ro-lima/documents/ publication/wcms_325664.pdf

6. Terraza H, Sturzenegger G. Dinámicas de Organización de los Recicladores Informales. Tres casos de estudio en América Latina [Internet]. Banco Interamericano de Desarrollo. 2010. Disponible en: http://www.iadb.org/en/publications/publicationdetail, $7101 . \mathrm{html}$ ?id=38366

7. Instituto Nacional de Estadistica e Informática. Producción y Empleo Informal en el Perú, Cuenta Satélite de la Economia Informal 2007-2012 [Internet]. 2014. Disponible en: http://www.inei.gob. pe/media/MenuRecursivo/publicaciones_digitales/ Est/Lib1154/libro.pdf

8. National Institute for Occupational Safety and Health (NIOSH). Cómo prevenir los trastornos musculoesqueléticos [Internet]. 2012. Disponible en: http://www.cdc.gov/spanish/niosh/docs/2012 120_sp/

9. Rojas M, Gimeno D, Vargas-Prada S, Benavides FG. Dolor musculoesquelético en trabajadores de América Central : resultados de la I Encuesta Centroamericana de Condiciones de Trabajo y Salud. Rev Panam Salud Pública [Internet]. 2015;38(2):120-8. Disponible en: http://iris.paho. org/xmlui/bitstream/handle/123456789/10046/ v38n2a04.pdf?sequence $=1$

10. Guillen-Subiran C. La relevancia del dolor crónico en el ámbito laboral. Gest Pract riesgos laborales. 2015;130:48-54.

11. nstituto Nacional de Rehabilitacion. Sala Situacional Primer Trimestre 2014 [Internet]. 2014. Disponible en: http://www.inr.gob.pe/transparencia/ Sala Situacional/2014/sala situacional i trimestre 2014.pdf

12. Ministerio de Trabajo y Promoción del Empleo. Anuario Estadístico Sectorial del MTPE 2014 [Internet]. 2015. Disponible en: http://www.trabajo.gob. pe/archivos/file/estadisticas/anuario/anuario_estadistico_2014.pdf

13. Instituto Nacional de Estadistica e Informática Una mirada a Lima Metropolitana [Internet]. INEI. 2014. p. 81. Disponible en: https://www.inei.gob. pe/media/MenuRecursivo/publicaciones_digitales/ Est/Lib1168/libro.pdf
14. Kuorinka I, Jonsson B, Kilbom A, Vinterberg $H$, Biering-Sørensen F, Andersson G, et al. Standardised Nordic questionnaires for the analysis of musculoskeletal symptoms. Appl Ergon [Internet]. 1987;18(3):233-7. Disponible en: http://www.ncbi. nlm.nih.gov/pubmed/15676628

15. Martinez B, Santo S, Bolea M, Casalod Y, Andres E. Validación del cuestionario nórdico musculoesquelético estandarizado en población española [Internet]. 12 th International Conference on Occupational Risk Prevention. Disponible en: http://www.prevencionintegral.com/en/canal-orp/ papers/orp-2014/validacion-cuestionario-nordicomusculoesqueletico-estandarizado-en-poblacionespanola

16. Crawford J. The Nordic Musculoskeletal Questionnaire. Occup Med (Chic III) [Internet]. 2007;57(4):300-1. Disponible en: http://occmed. oxfordjournals.org/content/57/4/300.short

17. Sobrino J. Migración interna y tamaño de localidad en México. Estud Demogr Urbanos Col Mex [Internet]. 2014;29(3(87)):443-79. Disponible en: http://www.redalyc.org/pdf/312/31235413001.pdf

18. Cervantes J, Palacios L. El trabajo en la pepena informal en México: nuevas realidades, nuevas desigualdades. Estud Demogr Urbanos Col Mex. 2012;27(1(79)):95-117.

19. Parizeau K. Urban political ecologies of informal recyclers' health in Buenos Aires, Argentina. Heal Place [Internet]. Elsevier; 2015;33:67-74. Disponible en: http://dx.doi.org/10.1016/j.healthplace.2015.02.007

20. Bazo ML, Sturion L, Probst vanessa S. Caracterização do reciclador da ONG RRV em Londrina-Paraná. Fisioter em Mov [Internet]. 2011;24(4):613-20. Disponible en: http://www. scielo.br/pdf/fm/v24n4/04.pdf

Artículo recibido el 4 de mayo de 2016 y aceptado para publicación el 7 de julio de 2016.

Financiamiento: Cofinanciado por la Universidad de Ciencias y Humanidades.

Conflicto de interés: No existe.

Correspondencia:

Juan Morales-Quispe.

Dirección: Av. Universitaria 5175, Los Olivos. Correo electrónico: mdjuanmorales@gmail.com 